\title{
Prognostic Significance of Microvessel Density Determining by Endoglin in Stage II Rectal Carcinoma: A Retrospective Analysis
}

\author{
Zeljko Martinovic, ${ }^{1}$ Drazen Kovac, ${ }^{2}$ and Mia Martinovic ${ }^{1}$ \\ ${ }^{1}$ Department of Surgery, Croatian Hospital "Dr. fra Mato Nikolic", 72276 Nova Bila, Bosnia and Herzegovina \\ ${ }^{2}$ Department of Pathology, School of Medicine, University of Rijeka, 51000 Rijeka, Croatia \\ Correspondence should be addressed to Zeljko Martinovic; zeljko.martinovic3@gmail.com
}

Received 17 February 2015; Revised 24 April 2015; Accepted 4 May 2015

Academic Editor: Hans J. Schmoll

Copyright (c) 2015 Zeljko Martinovic et al. This is an open access article distributed under the Creative Commons Attribution License, which permits unrestricted use, distribution, and reproduction in any medium, provided the original work is properly cited.

\begin{abstract}
Background. The role of endoglin in the Dukes B rectal cancer is still unexplored. The aim of this study was to examine the expression of endoglin (CD105) in resected rectal cancer and to evaluate the relationship between microvessels density (MVD), clinicopathological factors, and survival rates. Methods. The study included 95 primary rectal adenocarcinomas, corresponding to 67 adjacent and 73 distant normal mucosa specimens from surgical resection samples. Tumor specimens were paraffinembedded and immunohistochemical staining for the CD105 endothelial antigen was performed to count CD105-MVD. For exact measurement of the CD105-MVD used a computer-integrated system Alphelys Spot Browser 2 was used. Results. The intratumoral CD105-MVD was significantly higher compared with corresponding adjacent mucosa $(P<0.0001)$ and distant mucosa specimens $(P<0.0001)$. There was no significant difference in the CD105-MVD according to patients age, gender, tumor location, grade of differentiation, histological type, depth of tumor invasion, and tumor size. The overall survival rate was significantly higher in the low CD105-MVD group of patients than in the high CD105-MVD group of patients (log-rank test, $P=0.0406)$. Conclusion. CD105-assessed MVD could help to identify patients with possibility of poor survival in the group of stage II RC.
\end{abstract}

\section{Introduction}

According to International Agency for Research on Cancer (IARC), colorectal cancer is the third most common cancer and the fourth leading cause of cancer death worldwide [1]. In the European Union (EU), colorectal cancer is the third most common cancer site and the second most common cause of death from cancer [2]. In the Federation of Bosnia and Herzegovina, rectal cancer is the third most common form of cancer in men (16,5\%) and the eighth most common in women (10,0\%) [3]. Despite significant improvements in the treatment of primary rectal cancer achieved during the last two decades, the long-term outcome of affected patients is still poor $[4,5]$. The type of therapy for rectal cancer depends on the tumor location and stage. The pelvic localization of the rectum limits the possibility of radical surgical resection which increases the risk of poor overall prognosis [6]. The group of stage II rectal cancers (pT3pT4, N0, and M0) includes tumors with different pathological characteristics and variable clinical behavior whose outcomes differ greatly. In patients with stage II supplemental risk estimation is crucial for treatment [7]. The traditional method of stratifying patients with rectal cancer cannot fully predict individual clinical outcome in this group [8].

Tumor growth and its spread to adjacent tissues depend on its ability to stimulate angiogenesis. Angiogenesis consists of formation of new blood vessels from preexisting vasculature [9]. The studies have shown that the angiogenic potential of a tumor may be inferred from its vascularity measured in histological section [10]. The count of blood microvessels of the tumor, as shown in microvessel density, has been recognized as an indicator of malignant potential of the tumors and provides the ability to predict tumors recurrence and survival rate. Many studies have concluded that MVD is inversely related to survival in colorectal cancer [11].

Tumors promote angiogenesis by secreting proangiogenic factors. The transforming growth factor- $\beta$ (TGF- $\beta$ ) is 
a large family of cytokines that play a key role in tumor angiogenesis. Endoglin (CD105) is a type III TGF- $\beta$ coreceptor and it is overexpressed on tumor neovasculature [12]. Endoglin has been suggested to be the most suitable marker available to quantify tumor angiogenesis [13]. In studies, increased CD105 expression as determined by immunohistochemical staining was associated with shorter overall survival rates, but these findings have not always been confirmed [14]. Our study aimed to examine immunohistochemical expression of CD105 in stage II rectal carcinomas and to investigate a correlation between CD105-assessed MVD and clinicopathological variables and to analyze prognostic value of MVD in the overall survival.

\section{Methods}

2.1. Patients and Specimens. We studied 95 cases of primary rectal adenocarcinomas in stage II (T3-T4, N0, and M0) treated by complete surgical resection (R0) in a 5-year period at Clinic for Surgery, Clinical Hospital Center Rijeka, Croatia, from January 2002 to December 2006. The study included 95 primary rectal adenocarcinomas, 95 adjacent normal mucosa specimens, and 95 distant normal mucosa specimens from surgical resection samples. The adjacent and distant normal mucosa corresponding to the primary tumor from the same patients were taken from the margin of near and distant surgical resection. Tissue samples included in this study were retrieved from the archives of the Institute of Pathology School of Medicine of Rijeka, Croatia. The exclusion criteria were a synchronous tumor or tumors in another localisation in anamnesis, emergency surgery, preoperative radiotherapy or chemotherapy, perforation of bowel, and incomplete clinical data. The study was approved by the University of Rijeka Ethics Committee and patients signed informed consent.

All of the patients underwent radical low anterior or abdominoperineal rectum resection. All patients had confirmed rectal adenocarcinomas by histopathology and were staged according to the 7th edition of the American Joint Committee on Cancer (AJCC) Staging Manual [15]. The histological grading was classified according to the WHO (World Health Organization) classification [16]. The mean duration of follow-up was $54.7 \pm 23.1$ months (median duration, 60,0 months) after the operation for rectal cancer (RC). Survival data and cause of death of those who died during follow-up period were obtained from the Croatian Cancer Registry. Patient and tumour characteristic are presented in Table 1.

2.2. Immunohistochemistry. Immunohistochemical analysis was performed on formalin-fixed paraffin-embedded section. All tissue samples from RC and adjacent and distant mucosa were fixed in $10 \%$ buffered formalin and embedded in paraffin. We prepared $4 \mu \mathrm{m}$ thick serial sections which were deparaffinized in xylene, rehydrated in graded ethanol, and washed with phosphate-buffered saline. Endogenous peroxidase was inhibited with $3 \%$ hydrogen peroxide. Tissue sections were incubated for 30 minutes with the anti-CD105 primary monoclonal antibody (mouse anti-human, clone
TABLE 1: Clinical and pathologic characteristics of the rectal cancer samples.

\begin{tabular}{lc}
\hline Characteristics & $\begin{array}{c}\text { Number of } \\
\text { patients }\end{array}$ \\
\hline Total number & 95 \\
Age, median 69 years & \\
$\quad \leq 69$ & 49 \\
$>69$ & 46 \\
Gender & \\
Male & 61 \\
Female & 34 \\
Surgery & \\
Low anterior resection & 76 \\
Abdominoperineal resection & 19 \\
Tumor location & \\
Upper rectum & 23 \\
Middle rectum & 52 \\
Low rectum & 20 \\
Grade of differentiation & \\
G1 & 52 \\
G2 & 37 \\
G3 & 34 \\
Histologic type & 64 \\
Adenocarcinoma & \\
Adenocarcinoma with mucinous features & 13 \\
Depth of tumor invasion & \\
T3 & \\
T4a & \\
T4b & \\
Tumor size & \\
$\quad 4$ cm & \\
\hline
\end{tabular}

SN6h, Dako Corporation, Denmark) at a 1:10 dilution. Primary antibody binding site was visualized using a secondary antibody detection kit (Envision + kit; Dako, Denmark).

The staining was visualized with diaminobenzidine (DAB). Tissue sections were counterstained with hematoxylin. Brown staining for CD105 was considered positive. Distant normal mucosa free of tumor was used as positive controls and the primary antibody was replaced with phosphate-buffered saline solution for negative controls.

\subsection{Evaluation of Staining and of MVD by Computerized} Image Analysis. All slides stained with anti-CD105 were viewed and analyzed with Alphelys Spot Browser 2 integrated system, using a software controlled (Alphelys Spot Browser 2.4.4., France) stage positioning Nikon Eclipse 50i microscope mounted $1360 \times 1024$ resolution Microvision CFW-1310C digital camera. The slides were scanned at $\times 20$ magnification to identify "hot spots" (areas with the highest microvessel concentration) for the slides and then at $\times 200$ 
TABLE 2: CD105-MVD and clinicopathological factors in patients with rectal cancer.

\begin{tabular}{|c|c|c|c|}
\hline Variable & $\begin{array}{c}\text { Patients } \\
n(\%)\end{array}$ & $\begin{array}{c}\text { CD105-MVD } \\
\text { median }(95 \% \mathrm{CI})\end{array}$ & $P$ value \\
\hline Age, median 69 years & & & $0.268^{*}$ \\
\hline$\leq 69$ & $49(51.6)$ & $192.8(152.6-296.1)$ & \\
\hline$>69$ & $46(48.4)$ & $164.2(125.8-192.2)$ & \\
\hline Gender & & & $0.499^{*}$ \\
\hline Male & $61(64.2)$ & $171.5(124.7-206.0)$ & \\
\hline Female & $34(35.8)$ & $184.6(159.4-247.3)$ & \\
\hline Tumor location & & & $0.529^{* *}$ \\
\hline Upper rectum & $23(24.2)$ & $200.9(165.2-285.8)$ & \\
\hline Middle rectum & $52(54.7)$ & $178.9(136.2-240.5)$ & \\
\hline Low rectum & $20(21.1)$ & $125.8(106.2-194.9)$ & \\
\hline Grade of differentiation & & & $0.245^{* *}$ \\
\hline G1 & $55(57.9)$ & $171.5(127.0-192.3)$ & \\
\hline G2 & $34(35.8)$ & 191.7 (124.1-289.2) & \\
\hline G3 & $6(6.3)$ & $267.1(105.6-578.2)$ & \\
\hline Histologic type & & & $0.160^{*}$ \\
\hline Adenocarcinoma & $82(86.3)$ & $173.2(137.1-193.4)$ & \\
\hline Adenocarcinoma with mucinous features & $13(13.7)$ & $235.2(127.3-423.8)$ & \\
\hline Depth of tumor invasion & & & $0.202^{* *}$ \\
\hline $\mathrm{T} 3$ & $37(38.9)$ & $173.2(120.1-189.2)$ & \\
\hline $\mathrm{T} 4 \mathrm{a}$ & $42(44.2)$ & $205.0(151.1-315.0)$ & \\
\hline $\mathrm{T} 4 \mathrm{~b}$ & $16(16.9)$ & $151.1(95.4-292.7)$ & \\
\hline Tumor size & & & $0.775^{*}$ \\
\hline$\leq 4 \mathrm{~cm}$ & $64(67.4)$ & $175.6(131.4-197.8)$ & \\
\hline$>4 \mathrm{~cm}$ & $31(32.6)$ & $196.1(142.7-244.7)$ & \\
\hline
\end{tabular}

${ }^{*}$ Mann-Whitney $U$ test; ${ }^{* *}$ Kruskal-Wallis test.

magnification to create images for quantification scoring positive cells and MVD. Positive cells were counted in the tumor and adjacent and distant normal mucosa and presented as percentage of positive cells and MVD as number of microvessels in the histological field according to Weidner et al. [17]. The regions with the most intensive vascularization ("hot spots") were defined by scanning the entire tumor section at low magnification with a selection of four fields. The area of this histological field was $0,612 \mathrm{~mm}^{2}$. "Hot spots" were identified by two independent observers at $\times 20$ magnification. Semiquantitative expression levels of CD105 were classified according to the following criteria: cases with $<1 \%$ positive cells (negative staining), 1-25\% positive cells (weak staining), 26-50\% positive cells (moderate staining), and $>50 \%$ positive cells (strong staining) as described by Dassoulas et al. [18].

2.4. Statistical Analysis. Statistical analysis was performed using MedCalc version 14.8.1 (MedCalc Software bvba, Mariakerke, Belgium). Descriptive statistics and 95\% confidence intervals were calculated to describe data. The distribution of data was tested for normality using the Smirnov-Kolmogorov test. McNemar's test was applied to examine the significance of the differences in CD105 expression in tumor, adjacent mucosa, and distant normal mucosa. The Mann-Whitney $U$ test and Kruskal-Wallis tests were used to compare MVD among the clinicopathological variables. The receiver operating curve (ROC) approach was used to determine best-fitting cut-off for the CD105 expression and MVD in terms of the survival analysis [19]. Survival analysis was estimated by the Kaplan-Meier method and compared by the log-rank test. Prognostic factors of survival were identified by the use of the Cox proportional hazard regression. Differences at $P<0.05$ were considered significant.

\section{Results}

3.1. Patient Sample Classification. We assessed paraffinembedded specimens from tumors from 95 patients resected for RC. Clinicopathological characteristics of patients are summarized in Table 2. The median age at diagnosis was 69 years (range 15 to 85 years), 49 patients (51.6\%) were $\leq 69$ years of age, and 46 patients $(48.4 \%)$ were $>69$ years old. Sixty-one (64.2\%) were males and 34 (35.8\%) were females. In 23 patients $(24.2 \%)$, the tumor was located in the upper rectum, in $52(54.7 \%)$ in the middle rectum and in $20(21.1 \%)$ in the low rectum. According to grade of differentiation, 55 patients (57.9\%) were G1 (well differentiated), 34 (35.8\%) 


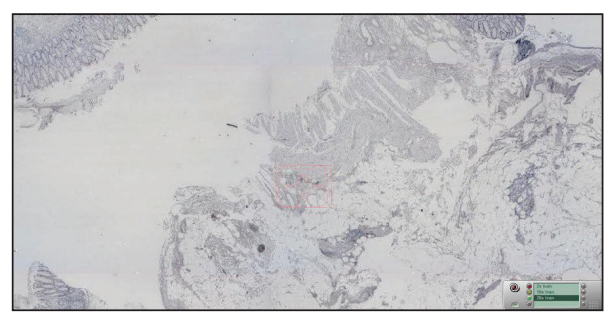

(a)

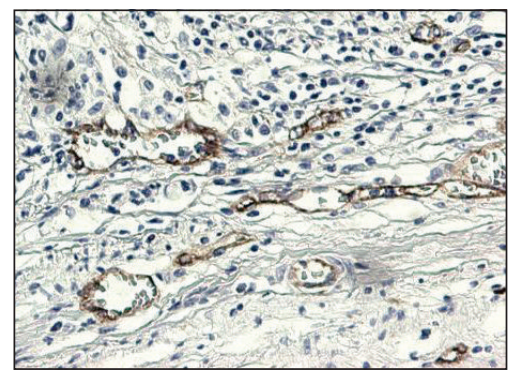

(b)

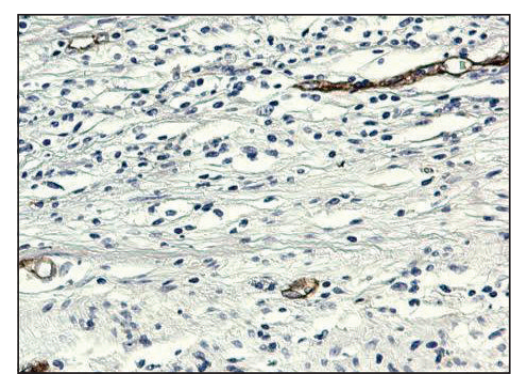

(c)

FIGURE 1: Immunohistochemical endoglin expression in rectal carcinoma (a), magnification $\times 20$. High endoglin expression (b) and low endoglin expression (c). Magnification $\times 200$.

G2 (moderately differentiated), and 6 (6.3\%) G3 (poorly differentiated). According to depth of tumor invasion, 37 patients (38.9\%) were T3, $42(44.2 \%) \mathrm{T} 4 \mathrm{a}$, and $16(16.9 \%)$ T4b. Eighty-two (86.3\%) tumors were classified as adenocarcinomas and $13(13.7 \%)$ as adenocarcinomas with mucinous features. Median tumor size was $3.8 \mathrm{~cm}$ (range, 1,3 to $12,0 \mathrm{~cm}$ ). The median patients follow-up was 60 months (range, 1.0 to 109.0 months). Of the 95 patients, 15 patients developed recurrent disease and 29 died of RC in the 5-year follow-up period.

\subsection{CD105 Expression in RC Samples. The CD105 (endoglin)} expression was analyzed by immunohistochemistry in the tumors, adjacent normal mucosa, and distant normal mucosa. The CD105 expression was detected on cell membrane of the endothelial cells in all the sites. Examples of low and high CD105 expression in the tumors are shown in Figure 1. Endoglin staining was observed in 93 of 95 tumours (97.9\%). Most specimens (71.6\%) had weak to moderate CD105 staining intensity, while $26.3 \%$ of specimens had strong staining intensity. Strong CD105 staining was found in $4(5.9 \%)$ samples of adjacent normal mucosa and in only one $(1.3 \%)$ sample of distant normal mucosa. Using a cutoff value of $\leq 48,8 \%$, tumors were divided into two groups: low CD105 expression and high CD105 expression. The count of the CD105 expression in the tumors, adjacent mucosa, and distant normal mucosa is shown in Figure 2. McNemar's test was performed. The CD105 expression in the tumor was significantly higher compared with the adjacent mucosa $(P=$ $0.001)$ and the distant normal mucosa $(P<0.001$; Figure 2$)$. There was no significant difference in CD105 expression in the adjacent mucosa and distant mucosa $(P=0.375)$.

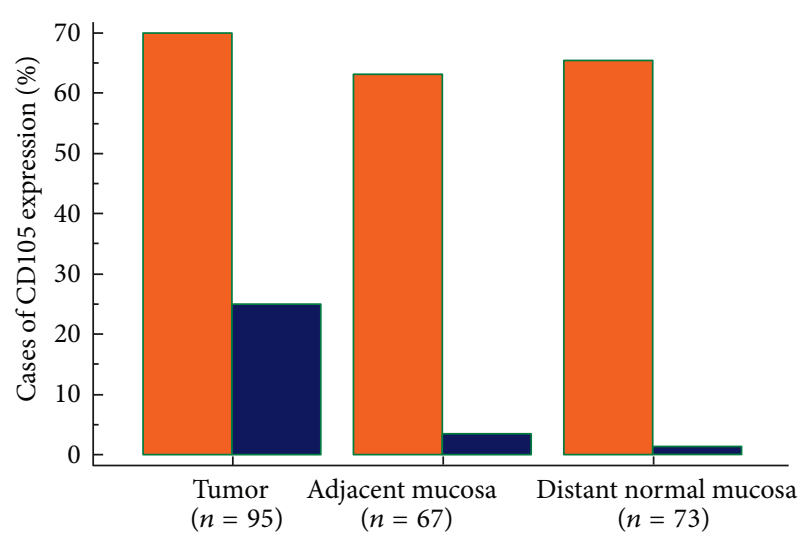

Low CD105 expression

High CD105 expression

FIgURE 2: The count of the CD105 expression in the tumour, adjacent normal mucosa, and distant normal mucosa. The count of the CD105 expression was significantly higher in the tumour compared with the adjacent normal mucosa $(P=0.0015)$ and the distant normal mucosa $(P<0.001)$.

3.3. Microvessel Density. Significant correlation between CD105 expression and MVD in tumors was determined by Spearman's coefficient of rank ( rho $=0.602, P<0.0001,95 \%$ CI 0.456 to 0.717 ). MVD were analyzed in tumors, adjacent mucosa, and distant mucosa. Median CD105-assessed MVD in tumors was 174.47 vessels $/ \mathrm{mm}^{2}$ (95\% CI 151.00-205.29), in adjacent mucosa 88.24 vessels $/ \mathrm{mm}^{2}$ (95\% CI 55.46-103.80), and in distant mucosa 58.82 vessels $/ \mathrm{mm}^{2}$ (95\% CI $51.42-$ 82.56). The MVD was significantly higher in the tumor 
TABLE 3: Overall survival rates and univariate analysis of patients with stage II rectal cancer.

\begin{tabular}{|c|c|c|c|}
\hline Variable & Number of patients & Overall survival rates & Log-rank test \\
\hline Age & & & 0.015 \\
\hline$\leq 69$ & 49 & 79.6 & \\
\hline$>69$ & 46 & 58.7 & \\
\hline Gender & & & 0.805 \\
\hline Male & 61 & 70.5 & \\
\hline Female & 34 & 67.6 & \\
\hline Tumor location & & & 0.446 \\
\hline Upper rectum & 23 & 69.6 & \\
\hline Middle rectum & 52 & 65.4 & \\
\hline Low rectum & 20 & 80.0 & \\
\hline Grade of differentiation & & & 0.721 \\
\hline G1 & 55 & 72.7 & \\
\hline G2 & 34 & 64.7 & \\
\hline G3 & 6 & 66.7 & \\
\hline Histologic type & & & 0.581 \\
\hline Adenocarcinoma & 82 & 68.3 & \\
\hline Adenocarcinoma with mucinous features & 13 & 76.9 & \\
\hline CD105 expression & & & 0.035 \\
\hline High & 24 & 87.5 & \\
\hline Low & 71 & 63.4 & \\
\hline CD105-MVD & & & 0.040 \\
\hline High & 44 & 79.5 & \\
\hline Low & 51 & 60.8 & \\
\hline
\end{tabular}

samples compared with adjacent mucosa $(P<0.0001)$ and the distant mucosa $(P<0.0001)$. There was no significant difference in the MVD according to patients age, gender, tumor location, grade of differentiation, histological tumor type, depth of tumor invasion, and tumor size (Table 2).

3.4. Univariate Survival Analysis. The Kaplan-Meier method and long-rang test were performed. There were significant differences in survival rates in the groups of patients with $\leq$ and $>69$ years old $(P=0.0156$; Table 3$)$. The group of patients with $\leq 69$ years of age had higher survival rate than patients $>69$ years of age. High CD105 expression was identified in $24(25,3 \%)$ of 95 cases and low or negative expression in 71 cases $(74,7 \%)$. CD105 expression showed a significant effect on patient survival (Figure 3). Patients with high CD105 expression had significantly longer overall survival time, compared to CD105 low expression group (logrank test, $P=0.035$ ).

The cut-off value for determining high and low MVD was performed by the receiver operating characteristic (ROC) curve analysis. The cut-off value was $\leq 179.7 \mathrm{microvessel} / \mathrm{mm}^{2}$ (sensitivity $69.0 \%$, specificity $53.0 \%$ ) (Figure 4). In a KaplanMeier survival estimate, the overall survival (OS) rate was significantly higher in the high MVD group of patients than the low MVD group of patients (Figure 5, log-rank test, $P=$ 0.040).
TABLE 4: The multivariate Cox proportional hazard regression analysis for survival in stage II rectal cancer patients (method "backward").

\begin{tabular}{|c|c|c|c|}
\hline Covariate & $P$ & OR & $(95 \% \mathrm{CI})$ \\
\hline Age $(\leq 69$ versus $>69)$ & 0.0110 & 1.0572 & $1.0130-1.1032$ \\
\hline $\begin{array}{l}\text { CD105 expression (low versus } \\
\text { high) }\end{array}$ & 0.0485 & 1.0373 & $1.0004-1.0754$ \\
\hline $\begin{array}{l}\text { CD105-MVD (low versus } \\
\text { high) }\end{array}$ & 0.0370 & 0.3156 & $0.1074-0.9275$ \\
\hline
\end{tabular}

Overall model fit $\chi^{2}=20.755, P=0.0004$.

MVD: microvessel density.

OR: odds ratio.

$95 \%$ CI: confidence interval.

3.5. Multiple Cox Regression Analysis. The prognostic variables were determined by Cox proportional hazard regression analysis. The full model containing all variables was statistically significant $(P<0.05)$, indicating that this model was able to distinguish between survival and nonsurvival. As shown in Table 4, three variables significantly affected the model, age, CD105 expression, and CD105-assessed MVD. "Backward" analysis was performed. The result showed that age $(\mathrm{OR}=1.05, P=0.0110), \mathrm{CD} 105$ expression $(\mathrm{OR}=$ 1.03, $P=0.0485)$, and CD105-assessed MVD (OR $=0.31$, $P=0.0370)$ were the independent prognostic factors for OS (Table 4). 


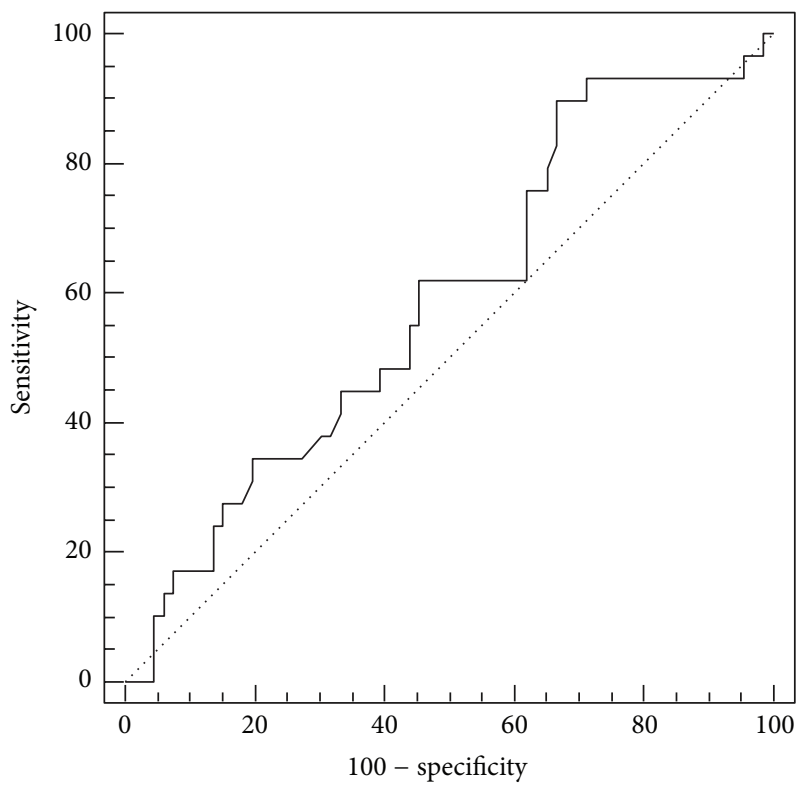

Sensitivity: 89.7

Specificity: 33.3

Criterion: $\leq 48.7$

FIGURE 3: Receiver operating characteristic (ROC) curve analysis for optimal cut-off point of CD105 expression. Using a cut-off value of $\leq 48,8 \%$, tumors were divided into two groups: low CD105 expression and high CD105 expression. ROC analysis indicated an optimal cut-off of $48,7 \%$ in prognostic survival in stage II rectal cancer. Area under the ROC curve (AUC): $0.586, P=0.171$.

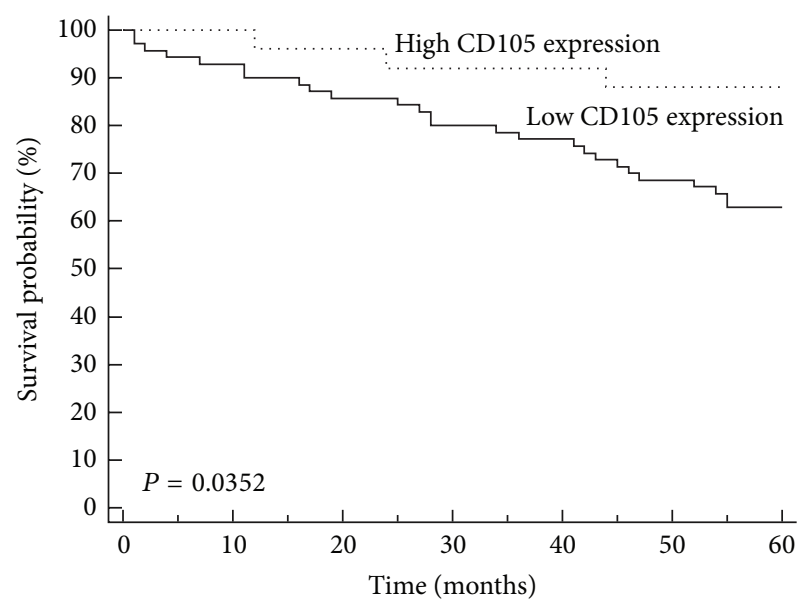

FIGURE 4: Kaplan-Meier analysis of overall survival for stage II rectal cancer patients according to low and high CD105 expression. CD105 expression shows significant correlation with survival of patients with stage II rectal carcinoma $(P=0.035)$.

\section{Discussion}

Stage II RC is defined by the presence of penetration through the muscularis propria and the absence of metastasis to either regional lymph nodes or distant sites [19]. However, death from RC of stage II continues to occur in approximately

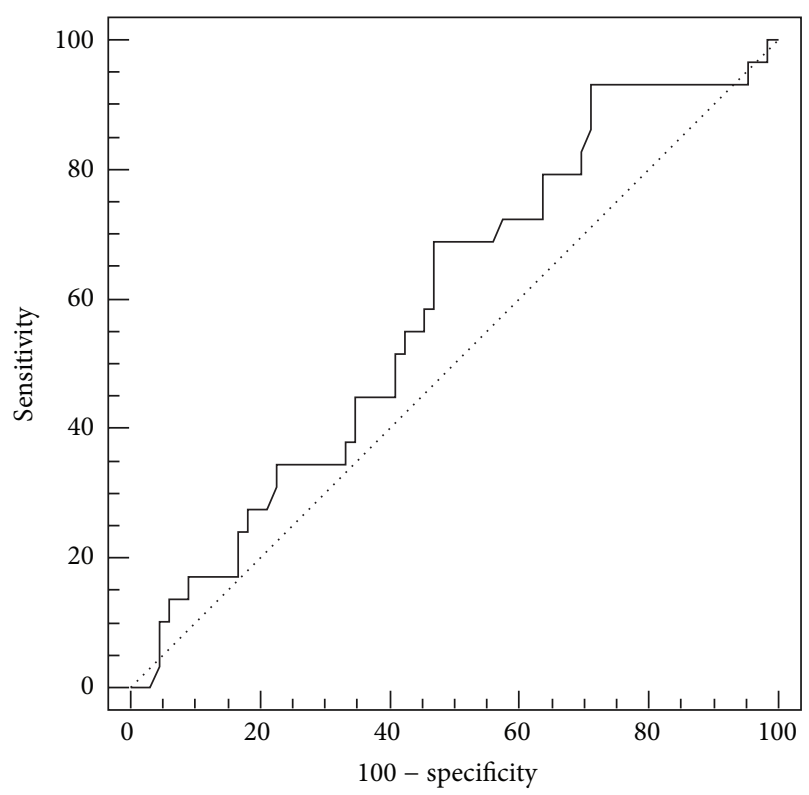

Sensitivity: 69.0

Specificity: 53.0

Criterion: $\leq 179.7$

FIGURE 5: Receiver operating characteristic (ROC) curve analysis for optimal cut-off point of CD105-MVD. According to the ROC curve analysis the hypervascularized tumours were discriminated from the hypovascularized tumours. ROC analysis indicated an optimal cut-off of $179.7 \mathrm{microvessel} / \mathrm{mm}^{2}$ in prognostic survival in stage II rectal cancer (Figure 6). Area under the ROC curve (AUC): 0.587, $P=0.165$.

$20 \%$ of patients $[20,21]$. Identifying high-risk patients with stage II RC is important because it my help to identify patients and additional risk for whom surgery alone may not be curative treatment. Endoglin is a proliferation-associated antigen on endothelial cells and essential for angiogenesis. It has been reported that expression of the endoglin in tumor endothelium may be a prognostic indicator of the outcome for various humans tumors including and colorectal cancer (CRC) [22]. These findings have not been confirmed by all researchers.

In the present study we analyzed the relationship between the CD105 expressions in RC, adjacent normal mucosa, and distant normal mucosa. In our cohort CD105 positive immunostaining was found in $97.9 \%$ of all rectal tumour specimens tested, with most samples having weak to moderate staining intensity (71.6\%). We showed that the CD105 expression levels significantly increase in RC from the distant and adjacent mucosa to the primary tumor (Figure 2). Endoglin was expressed at low level in endothelial cells in normal mucosa of the rectum and strongly expressed in vascular endothelial cells in tumor vessels. Our study has shown that CD105 is upregulated in RC tissue in comparison with normal mucosa of rectum. The intensity of staining for endoglin indicates that endoglin is a powerful marker of neovascularization in RC. This pattern of expression of 




Figure 6: Kaplan-Meier curve for patients with stage II rectal cancer according to microvessel density (MVD) by CD105. CD105 microvessel density correlates significantly with survival of patients with stage II rectal cancer (179.7 microvessels $/ \mathrm{mm}^{2}$, as the cut-off).

the endoglin shows its role as proangiogenic component in tumor endothelial cells.

Previous studies have demonstrated that increased endoglin expression assessed immunohistochemically correlates with the decreased survival period. Svagzdys et al. and Dassoulas et al. showed a clear association of cancerspecific OS with high CD105 expression [18, 23]. We also analyzed the relationship between the CD105 expression and patients survival. Our results showed that there was significant correlation between low CD105 expression and short survival in the cohort of our RC patients. Patients with high CD105 expression had a good prognosis.

Microvessel density assessment is the most commonly used technique to quantify intratumoral angiogenesis in cancer. In the present study, we assessed MVD with CD105 marker in RC tissue and adjacent and distant normal mucosa. Endoglin microvessel immunostaining was consistently present in all the cases studied. Consistent with previous studies, we found a significant increase of MVD in RC compared with their corresponding adjacent and distant normal mucosa $[24,25]$. These results support the role of CD105 as an optimal marker of proliferation of endothelial cells and its potential as prognostic factor $[13,14,26]$. The results of previous studies have shown that the use of CD105-MVD does not correlate with other histopathological parameters in the cohort of RC (Svagzdys et al.) or CRC (Dassoulas et al., Saad et al.) [18, 23, 27]. In our study, we also have not found a statistically significant correlation between CD105-MVD and conventional histopathological parameters in the cohort of stage II RC patients (Table 2).

Survival rates in our cohort stage II RC patients were analyzed according to age, gender, tumor location, grade of differentiation, histology, CD105 expression, and MVD. By univariate analysis, age, MVD, and CD105 expression were found to be significant prognostic factors for OS. In patients older than 69 years, low MVD and low CD105 expression were of high risk, with short median of OS. In our cohort, decreased CD105-MVD was associated with decreased OS. In multivariate analysis (Table 4 ) by the Cox proportional hazard regression model, age, CD105 expression, and CD105MVD had significance as independent prognostic factor for OS.

Patients with stage II RC have poor survival despite multimodality treatment [28]. Although angiogenesis affects the outcome of treatments, the importance of angiogenesis as a prognostic factor is still not clearly enough defined. In the studies, there are considerable differences in microvessel counts in tissue of rectal carcinoma. The quantification of microvessel density was made in the majority of studies with classical Weidner's method [17]. In our study, tumor microvessel density was obtained by computerized image analysis.

For colorectal cancer, conflicting results have been reported on the prognostic importance of MVD in various subsets of patients. Due to inconsistent methods of analysis of tumor angiogenesis in various studies, it is difficult to compare the values of MVD obtained in our analysis with the results of other authors. In our analysis, we found higher values of MVD (CD105-MVD, 221.0/ $\mathrm{mm}^{2}$ on average) in RC tissues in comparison with the results in the study of Svagzdys et al. (CD34-MVD, $193.0 / \mathrm{mm}^{2}$ on average), possibly due to the larger surface of the analyzed tumor tissue $\left(0.612 / \mathrm{mm}^{2}\right.$ versus $0.576 / \mathrm{mm}^{2}$ ) and the use of different endothelial cell markers [23]. In the present study, the microvessel counts are high and confirm that the rectal carcinoma is strongly dependent on angiogenesis.

Furthermore, significantly lower rates of survival were found in patients with lower MVD than cut-off value obtained by ROC analysis. This is shown by Kaplan-Meier survival curves for MVD variable (Figure 5). Our results suggest that the higher CD105-MVD accompanied by good OS, which is not in accordance with the results of most of the authors that a high MVD accompanied with poor OS [23]. Prall et al. have reported higher survival rate in tumors with high MVD (factor VIII immunostaining, $0.74 \mathrm{~mm}^{2}$, median as cut-off) which is consistent with our results [29]. According to the results of Uribarrena et al., patients with stages I and II colorectal carcinomas with higher vascularized tumor area had a significant association with a better outcome, but no significant relationship was observed between MVD and tumor recurrence and death [30]. Some studies demonstrated that high MVD counts determined using CD105 were strongly associated with a poor survival rate ( $\mathrm{Li}$ et al., median as cut-off) and high risk of metastatic disease (Saad et al., Romani et al., median as cut-off) [27, 31, 32].

However, the results of different studies linking lower MVD in tumors with poor survival and in various other solid tumors [33]. Endoglin is an auxiliary membrane receptor for transforming growth factor-beta (TGF- $\beta$ ) that modulates TGF- $\beta$ signaling [33]. Recently, endoglin has been identified as a key regulator of tumor cells proliferation, migration, and invasion $[24,33]$. Craft et al. showed that endoglin expression was lost during prostate cancer cell progression and that it led to increased cell invasion and migration [34]. It has been suggested that endoglin deficiency results 
in angiogenic adaptation, weakens the endothelial barrier, and increases metastatic spread, and may be associated with cancer progression [35].

\section{Conclusion}

In conclusion, this study showed that the CD105 expression and CD105-MVD are useful markers for identifying patients with an aggressive form of stage II RC. CD105-assessed MVD could help to identify patients with possibility of poor survival in the group of stage II RC.

\section{Conflict of Interests}

The authors declare that there is no conflict of interests regarding the publication of this paper.

\section{Acknowledgments}

The authors are grateful to Ozren Štanfel and Ivan Dašek for excellent technical assistance.

\section{References}

[1] J. Ferlay, I. Soerjomataram, R. Dikshit et al., "Cancer incidence and mortality worldwide: sources, methods and major patterns in GLOBOCAN 2012," International Journal of Cancer, vol. 136, no. 5, pp. E359-E386, 2015.

[2] J. Ferlay, E. Steliarova-Foucher, J. Lortet-Tieulent et al., "Cancer incidence and mortality patterns in Europe: estimates for 40 countries in 2012," European Journal of Cancer, vol. 49, no. 6, pp. 1374-1403, 2013.

[3] Cancer Registry Federation of Bosnia and Herzegovina, Overview of Malignantneoplasm, Public Health Institute of Federation of Bosnia and Herzegovina, 2011.

[4] E. Finlayson, "RAMPing up the quality of rectal cancer surgery," Journal of Clinical Oncology, vol. 32, no. 27, pp. 2938-2939, 2014.

[5] A. Vignali and P. de Nardi, "Multidisciplinary treatment of rectal cancer in 2014: where are we going?" World Journal of Gastroenterology, vol. 20, no. 32, pp. 11249-11261, 2014.

[6] R. Glynne-Jones, "Do T3 rectal cancer always need radiochemotherapy?" in Early Gastrointestinal Cancers II: Rectal Cancer, vol. 203 of Recent Results in Cancer Research, pp. 95-115, Springer, Berlin, Germany, 2014.

[7] N. I. Schneider and C. Langner, "Prognostic stratification of colorectal cancer patients: current perspectives," Cancer Management and Research, vol. 6, no. 2, pp. 291-300, 2014.

[8] S. F. Martins, R. M. Reis, A. M. Rodrigues, F. Baltazar, and A. L. Filho, "Role of endoglin and VEGF family expression in colorectal cancer prognosis and anti-angiogenic therapies," World Journal of Clinical Oncology, vol. 2, no. 6, pp. 272-280, 2011.

[9] R. Oklu, T. G. Walker, S. Wicky, and R. Hesketh, "Angiogenesis and current antiangiogenic strategies for the treatment of cancer," Journal of Vascular and Interventional Radiology, vol. 21, no. 12, pp. 1791-1805, 2010.

[10] G. Marioni, A. Staffieri, E. Manzato et al., "A higher CD105assessed microvessel density and worse prognosis in elderly patients with laryngeal carcinoma," Archives of Otolaryngology-Head \& Neck Surgery, vol. 137, no. 2, pp. 175-180, 2011.
[11] G. Des Guetz, B. Uzzan, P. Nicolas et al., "Microvessel density and VEGF expression are prognostic factors in colorectal cancer. Meta-analysis of the literature," British Journal of Cancer, vol. 94, no. 12, pp. 1823-1832, 2006.

[12] G. Bellone, C. Gramigni, B. Vizio et al., "Abnormal expression of Endoglin and its receptor complex (TGF- $\beta 1$ and TGF- $\beta$ receptor II) as early angiogenic switch indicator in premalignant lesions of the colon mucosa," International Journal of Oncology, vol. 37, no. 5, pp. 1153-1165, 2010.

[13] E. Fonsatti, H. J. M. Nicolay, M. Altomonte, A. Covre, and M. Maio, "Targeting cancer vasculature via endoglin/CD105: a novel antibody-based diagnostic and therapeutic strategy in solid tumours," Cardiovascular Research, vol. 86, no. 1, pp. 1219, 2010.

[14] C. Bernabeu, J. M. Lopez-Novoa, and M. Quintanilla, "The emerging role of TGF- $\beta$ superfamily coreceptors in cancer," Biochimica et Biophysica Acta, vol. 1792, no. 10, pp. 954-973, 2009.

[15] "Colon and rectum," in AJCC Cancer Staging Manual, B. Edge, D. R. Byrd, C. C. Compton, A. G. Fritz, F. L. Greene, and A. Trotti, Eds., pp. 143-164, Springer, New York, NY, USA, 7th edition, 2010.

[16] V. Barresi, L. R. Bonetti, A. Ieni, G. Branca, L. Baron, and G. Tuccari, "Histologic grading based on counting poorly differentiated clusters in preoperative biopsy predicts nodal involvement and pTNM stage in colorectal cancer patients," Human Pathology, vol. 45, no. 2, pp. 268-275, 2014.

[17] N. Weidner, J. P. Semple, W. R. Welch, and J. Folkman, "Tumor angiogenesis and metastasis-correlation in invasive breast carcinoma," The New England Journal of Medicine, vol. 324, no. 1, pp. 1-8, 1991.

[18] K. Dassoulas, M. Gazouli, G. Theodoropoulos et al., "Vascular endothelial growth factor and endoglin expression in colorectal cancer," Journal of Cancer Research and Clinical Oncology, vol. 136, no. 5, pp. 703-708, 2010.

[19] K. Sǿreide, "Receiver-operating characteristic curve analysis in diagnostic, prognostic and predictive biomarker research," Journal of Clinical Pathology, vol. 62, no. 1, pp. 1-5, 2009.

[20] C. C. Compton, "Optimal pathologic staging: defining stage II disease," Clinical Cancer Research, vol. 13, no. 22, pp. 6862s6870s, 2007.

[21] J. Zhu, Y. Xu, W. Gu et al., “Adjuvant therapy for T3N0 rectal cancer in the total mesorectal excision era- identification of the high risk patients," Radiation Oncology, vol. 5, article 118, 2010.

[22] F. Nassiri, M. D. Cusimano, B. W. Scheithauer et al., "Endoglin (CD105): a review of its role in angiogenesis and tumor diagnosis, progression and therapy," Anticancer Research, vol. 31, no. 6, pp. 2283-2289, 2011.

[23] S. Svagzdys, V. Lesauskaite, D. Pavalkis, I. Nedzelskiene, D. Pranys, and A. Tamelis, "Microvessel density as new prognostic marker after radiotherapy in rectal cancer," BMC Cancer, vol. 9, no. 3, article 95, 2009.

[24] U. Skoufi, D. L. Arvanitis, L. Lampri et al., "Association of claudin-1 with E- cadherin/catenin complex, microvessel density (MVD)-related markers, and clinicopathological features in colorectal carcinoma," Journal of Interdisciplinary Histopathology, vol. 2, no. 3, pp. 126-144, 2014.

[25] L. J. A. C. Hawinkels, P. Kuiper, E. Wiercinska et al., "Matrix metalloproteinase-14 (MT1-MMP)-mediated endoglin shedding inhibits tumor angiogenesis," Cancer Research, vol. 70, no. 10, pp. 4141-4150, 2010. 
[26] S. H. Fox, G. F. Whalen, M. M. Sanders et al., "Angiogenesis in normal tissue adjacent to colon cancer," Journal of Surgical Oncology, vol. 69, no. 4, pp. 230-234, 1998.

[27] R. S. Saad, Y. L. Liu, G. Nathan, J. Celebrezze, D. Medich, and J. F. Silverman, "Endoglin (CD105) and vascular endothelial growth factor as prognostic markers in colorectal cancer," Modern Pathology, vol. 17, no. 2, pp. 197-203, 2004.

[28] T. D. Wagner, M. G. Fakih, and G. Y. Yang, "Management of stage II/III rectal cancer," Journal of Gastrointestinal Oncology, vol. 1, no. 2, pp. 112-119, 2010.

[29] F. Prall, U. Gringmuth, H. Nizze, and M. Barten, "Microvessel densities and microvascular architecture in colorectal carcinomas and their liver metastases: significant correlation of high microvessel densities with better survival," Histopathology, vol. 42, no. 5, pp. 482-491, 2003.

[30] R. Uribarrena A, J. Ortego, J. Fuentes, N. Raventós, P. Parra, and R. Uribarrena E, "Prognostic value of microvascular density in dukes A and B (T1-T4, N0, M0) colorectal carcinomas," Gastroenterology Research and Practice, vol. 2009, Article ID 679830, 7 pages, 2009.

[31] C. Li, R. Gardy, B. K. Seon et al., "Both high intratumoral microvessel density determined using CD105 antibody and elevated plasma levels of CD105 in colorectal cancer patients correlate with poor prognosis," British Journal of Cancer, vol. 88, no. 9, pp. 1424-1431, 2003.

[32] A. A. Romani, A. F. Borghetti, P. Del Rio, M. Sianesi, and P. Soliani, "The risk of developing metastatic disease in colorectal cancer is related to CD105-positive vessel count," Journal of Surgical Oncology, vol. 93, no. 6, pp. 446-455, 2006.

[33] E. Pérez-Gómez, G. Del Castillo, J. F. Santibáñez, J. M. LópezNovoa, C. Bernabéu, and M. Quintanilla, "The role of the TGF$\beta$ coreceptor endoglin in cancer," TheScientificWorldJOURNAL, vol. 10, pp. 2367-2384, 2010.

[34] C. S. Craft, D. Romero, C. P. H. Vary, and R. C. Bergan, "Endoglin inhibits prostate cancer motility via activation of the ALK2-Smad1 pathway," Oncogene, vol. 26, no. 51, pp. 7240-7250, 2007.

[35] C. Anderberg, S. I. Cunha, Z. Zhai et al., "Deficiency for endoglin in tumor vasculature weakens the endothelial barrier to metastatic dissemination," The Journal of Experimental Medicine, vol. 210, no. 3, pp. 563-579, 2013. 


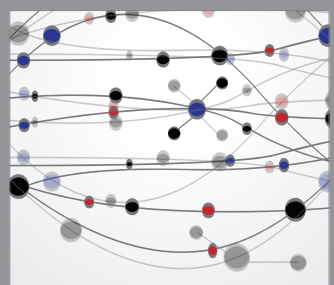

The Scientific World Journal
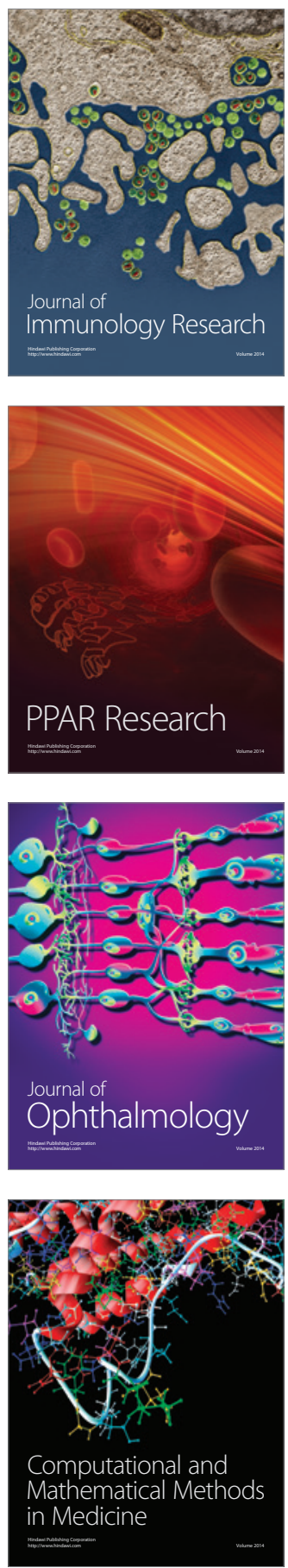

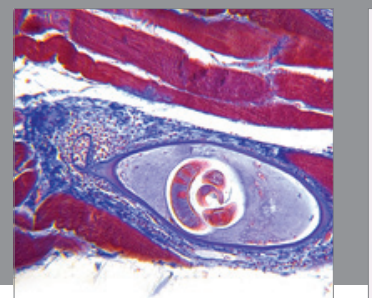

Gastroenterology

Research and Practice
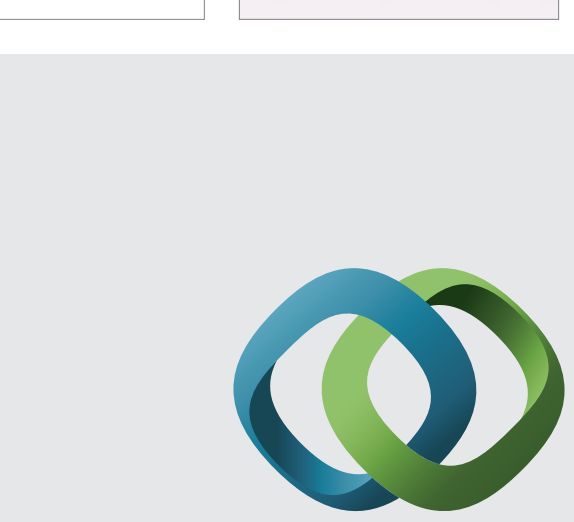

\section{Hindawi}

Submit your manuscripts at

http://www.hindawi.com
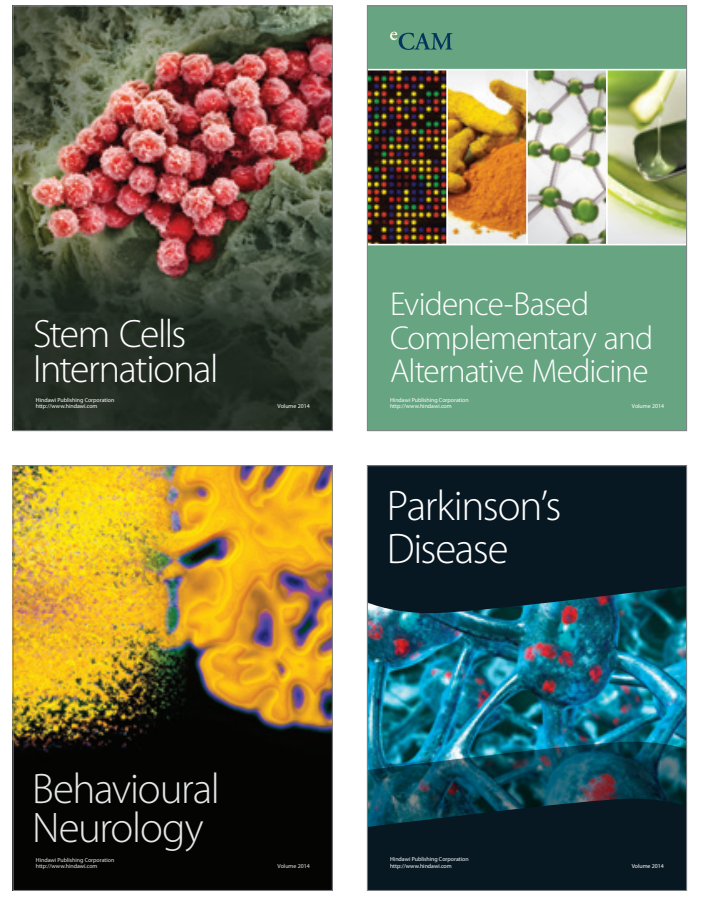
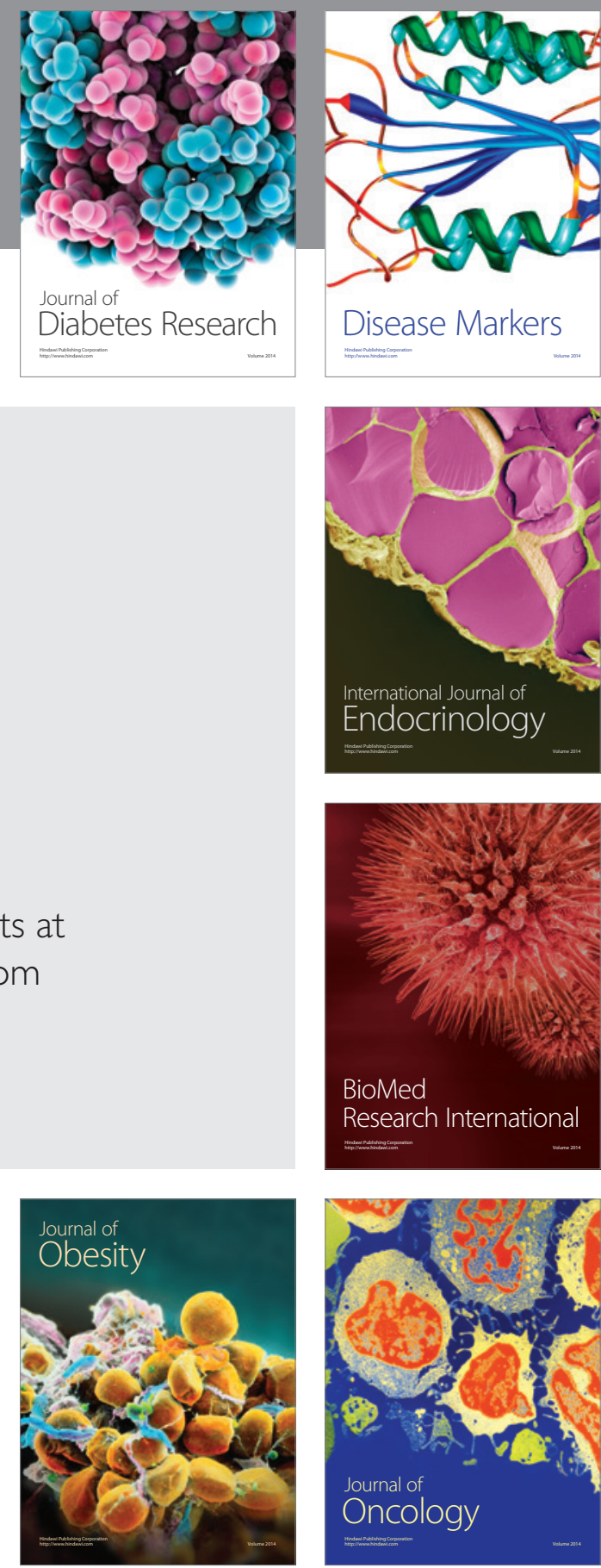

Disease Markers
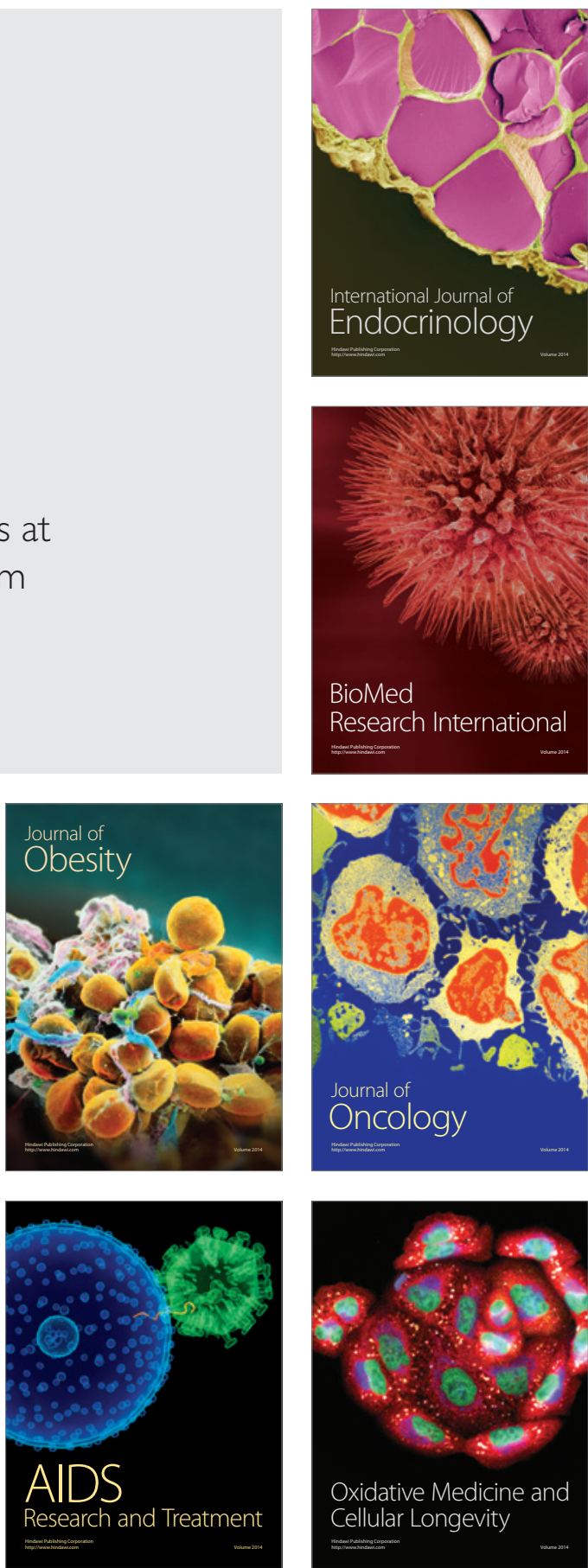